\title{
Helminths' antigens differentially modulate the activation of SARS-CoV-2-reactive helper and cytotoxic T cells in COVID-19 patients and healthy blood donors.
}

\author{
Tomabu Adjobimey ( $\sim$ Tomabu.Adjobimey@ukb.uni-bonn.de) \\ University Hospital Bonn: Universitatsklinikum Bonn https://orcid.org/0000-0002-4353-2897 \\ Julia Meyer \\ Vedrana Terkeš \\ Marijo Parcina \\ Achim Hoerauf
}

\section{Research Article}

Keywords: Helminths, immunomodulation, SARS-CoV-2-peptide pools (SPP), CD4/CD8 T cells

Posted Date: January 4th, 2022

DOI: https://doi.org/10.21203/rs.3.rs-1161617/v2

License: (c) (i) This work is licensed under a Creative Commons Attribution 4.0 International License.

Read Full License 


\section{Abstract \\ Background}

Contrary to the predictions, prevalence and mortality due to COVID-19 have remained moderate on the African continent. Several factors, including age, genetics, vaccines, and co-infections, might impact the course of the pandemic in Africa. Helminths are highly endemic in Sub-Saharan Africa and are renowned for their ability to modulate their host immune reactions.

\section{Methods}

Here we analyzed in vitro the impact of major helminth antigens on the immune reactivity to SARS-CoV-2 in COVID-19 patients using flow cytometry and Luminex.

\section{Results:}

We observed that helminth antigens significantly reduced the frequency of SARS-CoV-2-reactive CD4+ T helper cells. In contrast, the expression of SARS-CoV-2-reactive CD8+ T cells was not affected. In addition, stimulation with helminth antigens was associated with increased IL-10 and a reduction of IFNY and TNFa.

\section{Conclusion:}

Our data offer a plausible explanation for the moderate incidence of COVID-19 in Africa and support the hypothesis that helper T cell-mediated immune responses to SARS-CoV-2 are mitigated in the presence of helminth antigens, while virus-specific cytotoxic $T$ cell responses are maintained.

\section{Introduction}

The COVID-19 pandemic is with no doubt one of the major crises humanity is facing since World War II. As the pandemic started, WHO predicted millions of COVID-19-deaths in Africa. Contrary to the predictions, almost two years after the first cases, prevalence and mortality have remained surprisingly moderate on the continent (1). While this has been widely attributed to the younger average age on the continent or the lack of testing capacities, another potential factor might be the co-endemicity of helminths. Indeed, helminth infections, widely spread in Sub-Saharan Africa, are long-lived parasites and renowned for their ability to suppress inflammatory immune reactions to assure persistence in their hosts $(2,3)$. Helminths trigger a modified T helper $(T h) 2$ response, where pro-inflammatory components are balanced by regulatory mechanisms including regulatory $T$ and $B$ cells, anti-inflammatory cytokines (IL10, TGF- $\beta$ ), alternatively activated macrophages, and non-cytolytic antibodies (4-6). Clinical manifestations in SARS-CoV-2-infections range from asymptomatic to the devastating Acute Respiratory 
Distress Syndrome (ARDS), where the patients require invasive mechanical ventilation (7). These severe COVID-19 manifestations have been associated with immunological hyperreactivity characterized by a cytokine storm with high levels of pro-inflammatory cytokines like IL-2, IL-6, IFNy, and TNFa $(8,9)$. Previous data have demonstrated that helminths possess significant immunomodulatory properties that dampen harmful hyperinflammatory responses to viruses, bacteria, and other parasites (10-16).

In the context of SARS-CoV-2-infections, such a helminth-mediated immune modulation can impact the physiopathology of the disease (17). Recent reports suggest an inverse correlation between the incidence of COVID-19 and parasitic infections (18). The present study investigates in vitro the putative impact of helminth co-infection on the activation and proliferation of SARS-CoV-2-specific T cells in healthy individuals and COVID-19 patients. The findings are particularly relevant for developing countries known to be also endemic for helminth parasites.

\section{Material And Methods}

Study design and clinical characteristics of study participants

The study was conducted between November 2020 and January 2021 and is part of a larger survey. Patients were recruited in Wershofen (Germany) and Zagreb (Croatia). A total of 50 COVID-19 patients (29 men and 21 women) were included in the study. Ethical approval for the study was granted by local ethics committees. All included COVID-19 patients had been reported positive by SARS-CoV-2 RT-PCR in official COVID-19 testing centers. 3 (6\%) out of the 50 COVID-19 patients had been hospitalized during the acute phase of infection. The majority, 41 (82\%), had mild symptoms, whereas $6(12 \%)$ were fully asymptomatic (Table 1). Samples from healthy blood donors, kindly provided by the Haematology Department of the University Hospital of Bonn, were used as controls. Healthy blood donors were all negative at SARS-CoV-2 RT-PCR and serology and included 13 men and 17 women (mean age $=32.5$ \pm 12.37 ) (Table 1).

After sample collection, peripheral blood mononuclear cells (PBMCs) isolated from venous blood of patients and controls were stimulated with SARS-CoV-2 peptide pools in the presence or absence of antigen extracts from Onchocerca volvulus (OvAg), Brugia malayi (BmAg), or Ascaris lumbricoides (AlAg). Culture medium (Med) and helminth antigens alone were used as stimulation controls. After 24 hours of incubation, culture supernatants were collected for cytokines expression and cell pellets for FACS staining.

\section{Helminth antigen preparation}

Soluble extracts from adult worms of Onchocerca volvulus, Brugia malayi, and Ascaris lumbricoides were prepared as previously described (19). Briefly, 20 frozen adult worms were thawed and transferred to a Petri dish pre-filled with sterile PBS. Following several washes in PBS, worms were placed inside a glass mortar (VWR, Langenfeld, Germany). 3-5 ml of RPMI-medium were added, and worms were crushed until a homogenous solution was obtained. The extracts were then centrifuged for 10 minutes at $300 \mathrm{~g}$ and 
$4^{\circ} \mathrm{C}$ to remove insoluble material. Supernatants were transferred to a new tube. Protein concentrations were then measured using the Pierce Coomassie Plus (Bradford) Assay Kit (ThermoFisher Scientific, San Diego, CA, USA) according to the manufacturer's instructions. Aliquots were stored at $-80^{\circ} \mathrm{C}$ until use. The optimal concentration for cell stimulation was defined using a titration assay, and the endotoxin level was determined using the Pierce Limulus amoebocyte lysate (LAL) Chromogenic quantification kit (ThermoFisher Scientific). The endotoxin level was routinely below the detection limit of $0.1 \mathrm{EU} / \mathrm{ml}$.

\section{PBMC isolation}

PBMCs were isolated using a Ficoll gradient as previously described (6). In brief, $15 \mathrm{ml}$ of heparinized blood was diluted 1:2 in PBS (Gibco, Life Technologies, Carlsbad, USA), transferred on $15 \mathrm{ml}$ of Ficoll (PAN Biotech $\mathrm{GmbH}$, Aidenbach, Germany), and centrifuged for $20 \mathrm{~min}$ at $800 \mathrm{~g}, 4^{\circ} \mathrm{C}$ and without brake. Cell suspensions were then washed twice, $8 \mathrm{~min}$ at $300 \mathrm{~g}, 4^{\circ} \mathrm{C}$, and re-suspended in fetal bovine serum (FBS)-supplemented RPMI 1640 medium (Gibco). The cells were finally counted using the trypan blue (ThermoFisher Scientific) exclusion method and diluted for stimulation and culture. $100 \mu \mathrm{L}$ of cell suspension at $2 \times 10^{7}$ cells $/ \mathrm{ml}$ were plated in each well.

\section{Stimulation and cell culture}

To test the impact of helminth antigens on the immune reactivity to SARS-CoV-2, PBMCs from COVID-19 patients $(\mathrm{N}=50)$ were isolated and stimulated with $1 \mu \mathrm{g} / \mathrm{ml}(50 \mu \mathrm{l}$ working solution at $5 \mu \mathrm{g} / \mathrm{ml})$ of SARSCoV-2-Spike-protein-peptide-pools (SPP) (Miltenyi Biotech, Bergisch Gladbach, Germany), in the presence of culture medium or $10 \mu \mathrm{g} / \mathrm{ml}(50 \mu \mathrm{l}$ working solution at $50 \mu \mathrm{g} / \mathrm{ml})$ of Brugia malayi, Onchocerca volvulus or Ascaris lumbricoides extracts. The total volume for each well was adjusted to $250 \mu \mathrm{l}$ with culture medium ( $10 \% \mathrm{FCS}$ in RPMI). After 24 hours of culture (at $37^{\circ} \mathrm{C}, 5 \% \mathrm{CO}_{2}$ ), $200 \mu$ l supernatants were collected and frozen until use, and cells were harvested and prepared for FACS staining.

\section{FACS staining}

All reagents were obtained from ThermoFisher Scientific, and staining was performed according to the manufacturer's instructions. Briefly, cells were harvested and washed with FACS buffer (PBS/2\% FCS) at $300 \mathrm{~g}$ for $8 \mathrm{~min} .1 \times 10^{6}$ cells were then resuspended in $100 \mu \mathrm{l}$ of FACS buffer and blocked with $1 \mu \mathrm{l}$ of FCblock (ThermoFisher Scientific) for $15 \mathrm{~min} .5 \mu \mathrm{g} / 1 \times 10^{5}$ cells of anti-human CD3-PerCP (clone: S4.1-7D6), CD4-eF450 (clone: RPA-T4), CD8-FITC (clone: RPA-T8), CD69-APC (clone: FN50) and CD137-PE (clone: $4 B 4)$, were added and the cell suspension was incubated for $30 \mathrm{~min}$ at $4^{\circ} \mathrm{C}$. Cells were then washed twice with FACS buffer and fixed in $300 \mu \mathrm{l}$ PFA (4\%). To correct spectral overlaps, fluorescence compensation was done using UltraComp ebeads (ThermoFisher Scientific). Data were acquired using a Cytoflex-S flow cytometer (Beckmann Coulter, Krefeld, Germany) and analyzed with FlowJo v10 software (LLC, Ashland, OR, USA).

\section{Luminex assay}


To quantify cytokine levels in culture supernatants, ProcartaPlex Human Cytokines Panels (ThermoFisher Scientific) were used according to the manufacturer's instructions. Briefly, anti-IL-6, TNFa, IFNY, and IL-10coated magnetic beads were incubated with $25 \mu$ of assay buffer, kit standards, or diluted supernatant (1:2) for 1 hour. $25 \mu \mathrm{l}$ of biotin-labeled detection antibodies mix was then added. The plates were incubated on an orbital shaker (Stuart, Staffordshire, UK) at $500 \mathrm{rpm}$ for $30 \mathrm{~min}$, and $50 \mu \mathrm{l}$ of diluted Streptavidin-PE was added. Plates were then incubated for an additional 30 minutes and washed using a hand-held magnetic plate washer. Afterward, the beads were re-suspended in $120 \mu \mathrm{l}$ reading buffer. Data were then acquired using a MAGPIX Luminex system and analyzed with ProcartaPlex Analyst software 1.0 (ThermoFisher Scientific).

\section{Ethics statement}

Each volunteer recruited for the study gave informed consent to participate. Ethics approval for the study was obtained from the ethical boards of the University Hospital Bonn (Lfd.Nr.439/20) and the Zadar General Hospital (02-3534/20-2/20).

\section{Statistics}

All graphs were generated using GraphPad Prism 8 (La Jolla, CA, USA). For cell activation data, P values were calculated using the Kruskal-Wallis test. Dunn's multiple comparison test was used to compare all settings. Two-way ANOVA and Tukey's multiple comparison tests were used to compare the modulation of T cell reactivity in healthy donors and COVID-19 patients. Asterisks indicate the level of significance: ns= non-significant; $*=P \leq 0.05 ; * *=P \leq 0.01 ; * * *=P \leq 0.001, * * \star *=P \leq 0.0001$. Significance is accepted if $\mathrm{P}<0.05$. For cytokine data, Student's t-test was used for comparisons.

\section{Results}

Modulation of CD4+ and CD8+ T cells by helminth antigens in COVID-19 patients

We identified SARS-CoV-2 reactive-T CD4+ and CD8+ cells in COVID-19 patients and healthy donors, as described elsewhere $(20,21)$. After co-stimulation with SPP and helminth antigens, we observed that all helminth antigens tested separately significantly reduced the expression of SARS-CoV-2-reactive CD4+ T helper cells (Fig. 1A). In contrast, the frequency of SARS-CoV-2-reactive CD8+ T cells was not affected by the presence of helminth antigens (Fig. 1B).

\section{Figure 1: Modulation SARS-CoV-2-reactive CD4+ T CD8+T cells by helminth antigens in COVID-19}

patients. Graphs indicate the frequency of CD69+, CD137+ in CD4+ (A) or CD8+(B) T cell populations. Each symbol represents individual donors. Bars indicate Means \pm SEM of the percentage of SARS-CoV-2reactive $T$ cells in each setting. Data were obtained from 50 COVID-19 patients. $P$ values were calculated using Kruskal-Wallis 'test followed by Dunn's multiple comparison post hoc. Asterisks indicate the level of significance: $n s=$ non-significant; $*=P \leq 0.05 ; * \star=P \leq 0.01 ; * \star \star=P \leq 0.001, * \star \star *=P \leq 0.0001$. Significance is accepted if $P<0.05$. 
To explore the mechanisms associated with this immune suppression by helminth antigens, we analyzed cytokine expression in culture supernatants using a Luminex-based multiplex immunoassay. Indeed, in wells stimulated with SPP in the presence of OvAg, BmAg, or AlAg, significantly higher levels of IL-10 were detected compared to wells stimulated SPP alone (Fig. 2A-C). Interestingly, while a non-significant increase of IL- 6 was seen in the presence of OvAg and BmAg, significance was reached in the presence of AlAg (Fig. 2D-F). Moreover, a significant reduction of SPP-induced IFNY and TNFa expression was seen in the presence of OvAg or BmAg, while a robust trend was observed in the presence of AlAg (Fig. 2G-L).

Figure 2: Suppression of SARS-CoV-2 reactive CD4+ T cells by helminth antigens correlated with an increase of IL-10 production. Graphs represent cytokine levels of 3 independent experiments, and bars represent means \pm SEM of cytokine expression - medium control. Data were obtained from 50 COVID-19 patients. Indicated $p$ values were calculated using Student's t-test. Significance is accepted if $P<0.05$.

Helminth antigens suppressed CD4+ T cells in both COVID-19 patients and healthy donors

We next compared the modulation of reactive T cells in healthy donors and COVID-19 patients. While the frequency of SARS-CoV-2 reactive CD4+ T cells was significantly higher in COVID-19 patients compared to healthy donors, all helminth antigens suppressed SARS-CoV-2-induced CD4+ activation T cells activation in both COVID-19 patients and healthy donors (Fig. 3).

Figure 3: Inhibition of SARS-CoV-2-reactive CD4+ by Ov, Bm, and Al antigens in healthy donors and COVID-19 patients. Graphs indicate the frequency of CD69+, CD137+ in CD4+ T cell populations. Each symbol represents individual donors. Data were obtained from 50 COVID-19 patients and 30 healthy donors. Blue circles and red squares represent the frequencies of SARS-CoV-2-reactive CD4+ cells in healthy donors and COVID-19 patients, respectively. Bars indicate Means \pm SEM of the percentage of SARS-CoV-2-reactive T cells in each setting. $P$ values were calculated using two-way ANOVA and Tukey's multiple comparison test. Asterisks indicate the level of significance: ns $=$ non-significant; ${ }^{*}=P \leq 0.05 ; * *=$ $P \leq 0.01 ; \star \star \star=P \leq 0.001, * \star \star \star=P \leq 0.0001$. Significance is accepted if $P<0.05$.

No significant impact of helminth antigens on SARS-CoV-2 reactive CD8+ T cells in both COVID-19 patients and healthy donors

We next analyzed the modulation of reactive CD8+ T cells in healthy donors and COVID-19 patients in the presence of the helminth antigens. In contrast to CD4+ T cells, the expression of SARS-CoV-2 reactive CD8+ $T$ cells was not significant in healthy donors compared to the unstimulated control. Nonetheless, CD8+ $T$ cells activation was not impacted by helminth antigens in both healthy donors and COVID-19 patients. No significant difference was seen in the frequency of SARS-CoV-2 reactive CD8+ T cells in wells stimulated with helminth antigens compared to culture medium on the one hand, and SPP and SPP + OvAg, BmAg or AlAg on the other (Fig. 4). 
Figure 4: No suppression of SARS-CoV-2-reactive CD8+ T cells by Ov, Bm, and Al antigens in healthy donors and COVID-19 patients. Graphs indicate the frequency of CD69+, CD137+ in CD8+ T cell populations. Each symbol represents individual donors. Data were obtained from 50 COVID-19 patients and 30 healthy donors. Blue circles and red squares represent the frequencies of SARS-CoV-2-reactive CD8+ cells in healthy donors and COVID-19 patients, respectively. $P$ values were calculated using ordinary two-way ANOVA and Tukey's multiple comparison test. Asterisks indicate the level of significance: ns= non-significant; $*=P \leq 0.05 ; * \star=P \leq 0.01 ; * \star \star=P \leq 0.001, * \star \star \star=P \leq 0.0001$. Significance is accepted if $P$ $<0.05$.

\section{Discussion}

Our data support the hypothesis that in the presence of helminth antigens, immune responses to SARSCoV-2 are mitigated with regards to CD4+ T-cells and pro-inflammatory cytokine responses. In contrast, SARS-CoV-2-reactive CD8+ T cell responses are maintained in COVID-19 patients. This may critically shift the overall immune response to SARS-CoV-2 such that overreaction and severe COVID-19 are avoided, similar to helminths' ability to mitigate systemic inflammatory response syndrome in animal models and in humans $(22,23)$. Our data also confirm hypotheses formulated by others, suggesting that helminth parasites and their derivatives might exert an anti-pathological effect in COVID-19 patients (24) and contradict speculations staging helminths as potential potentiators of COVID-19 related morbidity and mortality through suppression of efficient immune response against SARS-CoV-2 $(25,26)$. Indeed, despite the implication of Th2 responses in the cytokine storm responsible for the pathology of COVID-19 (26), the typical helminth-induced Th2 immune response is associated with a strong regulatory arm that might significantly mitigate the disease severity $(4,5)$. The maintenance of SARS-CoV-2 specific cytotoxic $T$ cells observed in COVID-19 patients in our study may be the key element that facilitates the elimination of SARS-CoV-2-infected cells.

The major limitation of the present study is the fact that all samples were collected in Europe. It cannot be excluded that samples from helminth endemic regions react differently. However, due to the pandemic, sample collection in helminth endemic countries was strongly compromised. Additional investigations are required to confirm these data in helminth endemic regions. Nonetheless, the data offer a plausible explanation for why African countries endemic for helminth parasites are moderately affected by the COVID-19 pandemic (1). These data may also be relevant to the immunogenicity of current COVID-19 vaccines in endemic helminth regions. Indeed, it is unclear if helminths 'induced immunomodulation, as recently hypothesized by Chacin-Bonilla et al., would not affect the efficacy of COVID-19 vaccines in helminth endemic regions (26).

\section{Conclusion}

The present study uniquely demonstrates that antigens from three major helminth parasites (Brugia malayi, Onchocerca volvulus, and Ascaris lumbricoides) could potentially modulate the immune reactivity to SARS-CoV-2 peptides by suppressing reactive CD4+ helper T cells while maintaining SARS-CoV-2 
reactive CD8+ cytotoxic T cells. This finding offers a plausible explanation for the moderate incidence of COVID-19 in helminth endemic countries and opens new research avenues into the impact of helminthinduced immune-regulation on the COVID-19 disease outcome and vaccination effectiveness in tropical countries.

\section{Abbreviations}

COVID-19: Coronavirus Disease 2019; SARS-CoV-2: Severe Acute Respiratory Syndrome Coronavirus-2 IL-10: Interleukin-10; IFNY: Interferon-ү; TNFa: Tumour necrosis factor-alpha; PBMC: Peripheral blood mononuclear cells; SPP: SARS-CoV-2-Spike-protein-peptide-pools.

\section{Declarations}

\section{Role of funding source and ethics committee approval}

The funders had no role in study design, data collection, analysis, decision to publish, or manuscript preparation.

Achim Hoerauf is funded by the Deutsche Forschungsgemeinschaft (DFG, German Research Foundation) under Germany's Excellence Strategy - EXC2151 - 390873048".

Ethical clearance for the study was given by the University Hospital Bonn's ethics committee and the ethical board of the General Hospital Zadar.

\section{Authors' contributions}

Study design: Achim Hoerauf, Tomabu Adjobimey.

Samples and clinical data collection and analysis: Marijo Parcina.

Data analysis, interpretation, and verification: Achim Hoerauf, Tomabu Adjobimey.

Paper writing: Achim Hoerauf, Tomabu Adjobimey.

\section{Declaration of interests}

The authors have declared that no competing interests exist. 


\section{Availability of data and materials}

The datasets used during the current study are available from the corresponding author on request.

\section{References}

1. Mbow M, Lell B, Jochems SP, Cisse B, Mboup S, Dewals BG, et al. COVID-19 in Africa: Dampening the storm? Science. 2020;369(6504):624-6.

2. Harris NL, Loke P. Recent Advances in Type-2-Cell-Mediated Immunity: Insights from Helminth Infection. Immunity. 2018;48(2):396.

3. nick.white@covid19crc.org C-CRCEa. Global coalition to accelerate COVID-19 clinical research in resource-limited settings. Lancet. 2020;395(10233):1322-5.

4. Maizels RM, McSorley HJ. Regulation of the host immune system by helminth parasites. J Allergy Clin Immunol. 2016;138(3):666-75.

5. Adjobimey T, Hoerauf A. Induction of immunoglobulin G4 in human filariasis: an indicator of immunoregulation. Ann Trop Med Parasitol. 2010;104(6):455-64.

6. Satoguina JS, Adjobimey T, Arndts K, Hoch J, Oldenburg J, Layland LE, et al. Tr1 and naturally occurring regulatory T cells induce IgG4 in B cells through GITR/GITR-L interaction, IL-10 and TGFbeta. Eur J Immunol. 2008;38(11):3101-13.

7. Badraoui R, Alrashedi MM, El-May MV, Bardakci F. Acute respiratory distress syndrome: a life threatening associated complication of SARS-CoV-2 infection inducing COVID-19. J Biomol Struct Dyn. 2020:1-10.

8. Melo AKG, Milby KM, Caparroz A, Pinto A, Santos RRP, Rocha AP, et al. Biomarkers of cytokine storm as red flags for severe and fatal COVID-19 cases: A living systematic review and meta-analysis. PLoS One. 2021;16(6):e0253894.

9. Mehta P, Fajgenbaum DC. Is severe COVID-19 a cytokine storm syndrome: a hyperinflammatory debate. Curr Opin Rheumatol. 2021.

10. Arndts K, Deininger S, Specht S, Klarmann U, Mand S, Adjobimey T, et al. Elevated adaptive immune responses are associated with latent infections of Wuchereria bancrofti. PLoS Negl Trop Dis. 2012;6(4):e1611.

11. Ritter M, Osei-Mensah J, Debrah LB, Kwarteng A, Mubarik Y, Debrah AY, et al. Wuchereria bancroftiinfected individuals harbor distinct IL-10-producing regulatory $B$ and $T$ cell subsets which are affected by anti-filarial treatment. PLOS Negl TropDis. 2019;13(5):e0007436.

12. Ritter M, Ndongmo WPC, Njouendou AJ, Nghochuzie NN, Nchang LC, Tayong DB, et al. Mansonella perstans microfilaremic individuals are characterized by enhanced type 2 helper $\mathrm{T}$ and regulatory $\mathrm{T}$ and $B$ cell subsets and dampened systemic innate and adaptive immune responses. PLoS Negl Trop Dis. 2018;12(1):e0006184. 
13. Kroidl I, Chachage M, Mnkai J, Nsojo A, Berninghoff M, Verweij JJ, et al. Wuchereria bancrofti infection is linked to systemic activation of CD4 and CD8 T cells. PLoS Negl Trop Dis. 2019;13(8):e0007623.

14. Hübner MP, Layland LE, Hoerauf A. Helminths and their implication in sepsis - a new branch of their immunomodulatory behaviour? Pathog Dis. 2013;69(2):127-41.

15. Loffredo-Verde E, Bhattacharjee S, Malo A, Festag J, Kosinska AD, Ringelhan M, et al. Dynamic, helminth-induced immune modulation influences the outcome of acute and chronic hepatitis $B$ virus infection. J Infect Dis. 2020;221(9):1448-61.

16. Colombe S, Machemba R, Mtenga B, Lutonja P, Kalluvya SE, de Dood CJ, et al. Impact of schistosome infection on long-term HIV/AIDS outcomes. PLoS Negl Trop Dis. 2018;12(7):e0006613.

17. Bradbury RS, Piedrafita D, Greenhill A, Mahanty S. Will helminth co-infection modulate COVID-19 severity in endemic regions? Nat Rev Immunol. 2020.

18. Gutman JR, Lucchi NW, Cantey PT, Steinhardt LC, Samuels AM, Kamb ML, et al. Malaria and Parasitic Neglected Tropical Diseases: Potential Syndemics with COVID-19? Am J Trop Med Hyg. 2020;103(2):572-7.

19. Prodjinotho UF, von Horn C, Debrah AY, Batsa Debrah L, Albers A, Layland LE, et al. Pathological manifestations in lymphatic filariasis correlate with lack of inhibitory properties of IgG4 antibodies on IgE-activated granulocytes. PLoS Negl Trop Dis. 2017;11(7):e0005777.

20. Mateus J, Grifoni A, Tarke A, Sidney J, Ramirez SI, Dan JM, et al. Selective and cross-reactive SARSCoV-2 T cell epitopes in unexposed humans. Science. 2020.

21. Braun J, Loyal L, Frentsch M, Wendisch D, Georg P, Kurth F, et al. SARS-CoV-2-reactive T cells in healthy donors and patients with COVID-19. Nature. 2020;587(7833):270-4.

22. Gondorf F, Berbudi A, Buerfent BC, Ajendra J, Bloemker D, Specht S, et al. Chronic filarial infection provides protection against bacterial sepsis by functionally reprogramming macrophages. PLoS Pathog. 2015;11(1):e1004616.

23. Panda M, Sahoo PK, Mohapatra AD, Dutta S, Thatoi PK, Tripathy R, et al. Decreased prevalence of sepsis but not mild or severe $P$. falciparum malaria is associated with pre-existing filarial infection. Parasit Vectors. 2013;6:203.

24. Siles-Lucas M, Gonzalez-Miguel J, Geller R, Sanjuan R, Perez-Arevalo J, Martinez-Moreno A. Potential Influence of Helminth Molecules on COVID-19 Pathology. Trends Parasitol. 2021;37(1):11-4.

25. Abdoli A. Helminths and COVID-19 Co-Infections: A Neglected Critical Challenge. ACS Pharmacol TransI Sci. 2020;3(5):1039-41.

26. Chacin-Bonilla L, Chacon-Fonseca N, Rodriguez-Morales AJ. Emerging issues in COVID-19 vaccination in tropical areas: Impact of the immune response against helminths in endemic areas. Travel Med Infect Dis. 2021;42:102087.

\section{Figures}


A

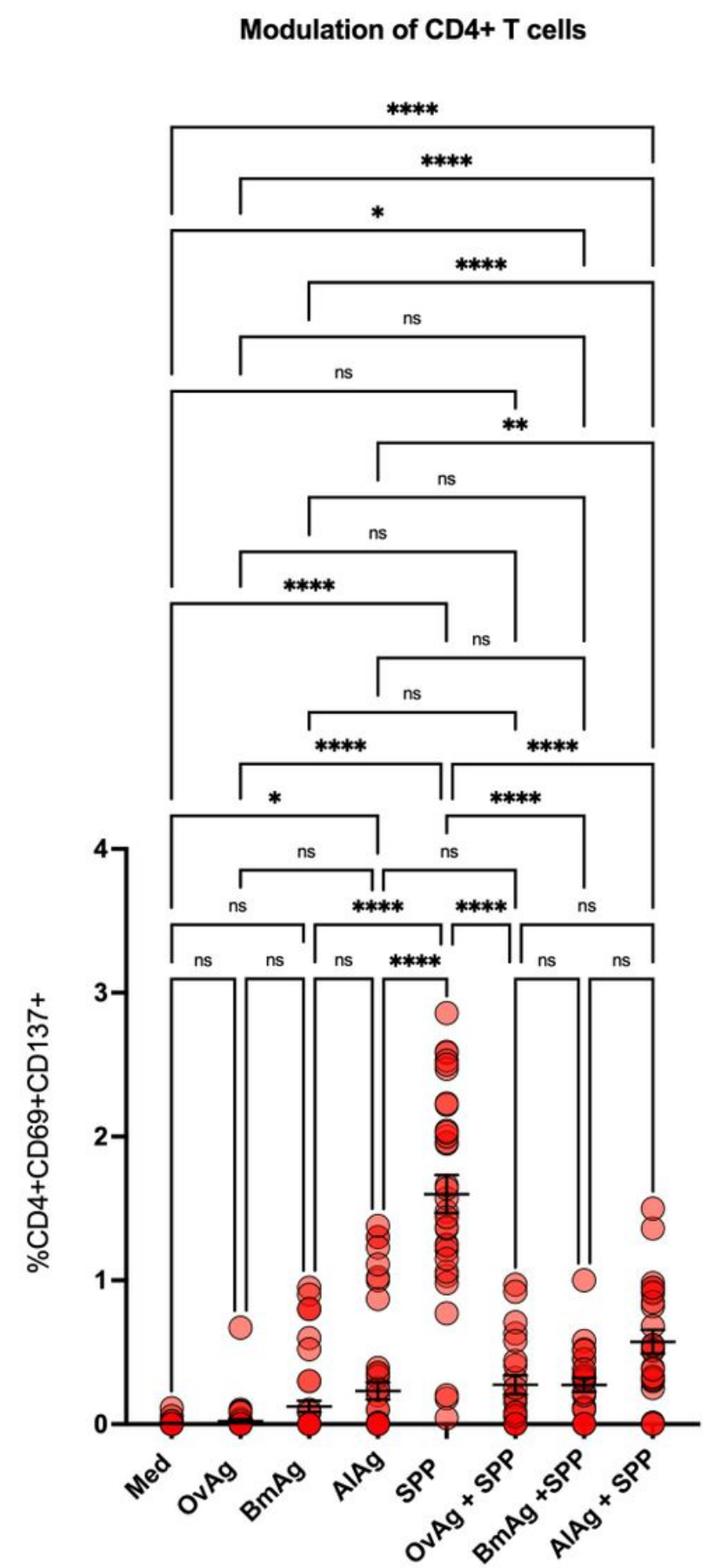

B

Modulation of $\mathrm{CD} 8+\mathrm{T}$ cells

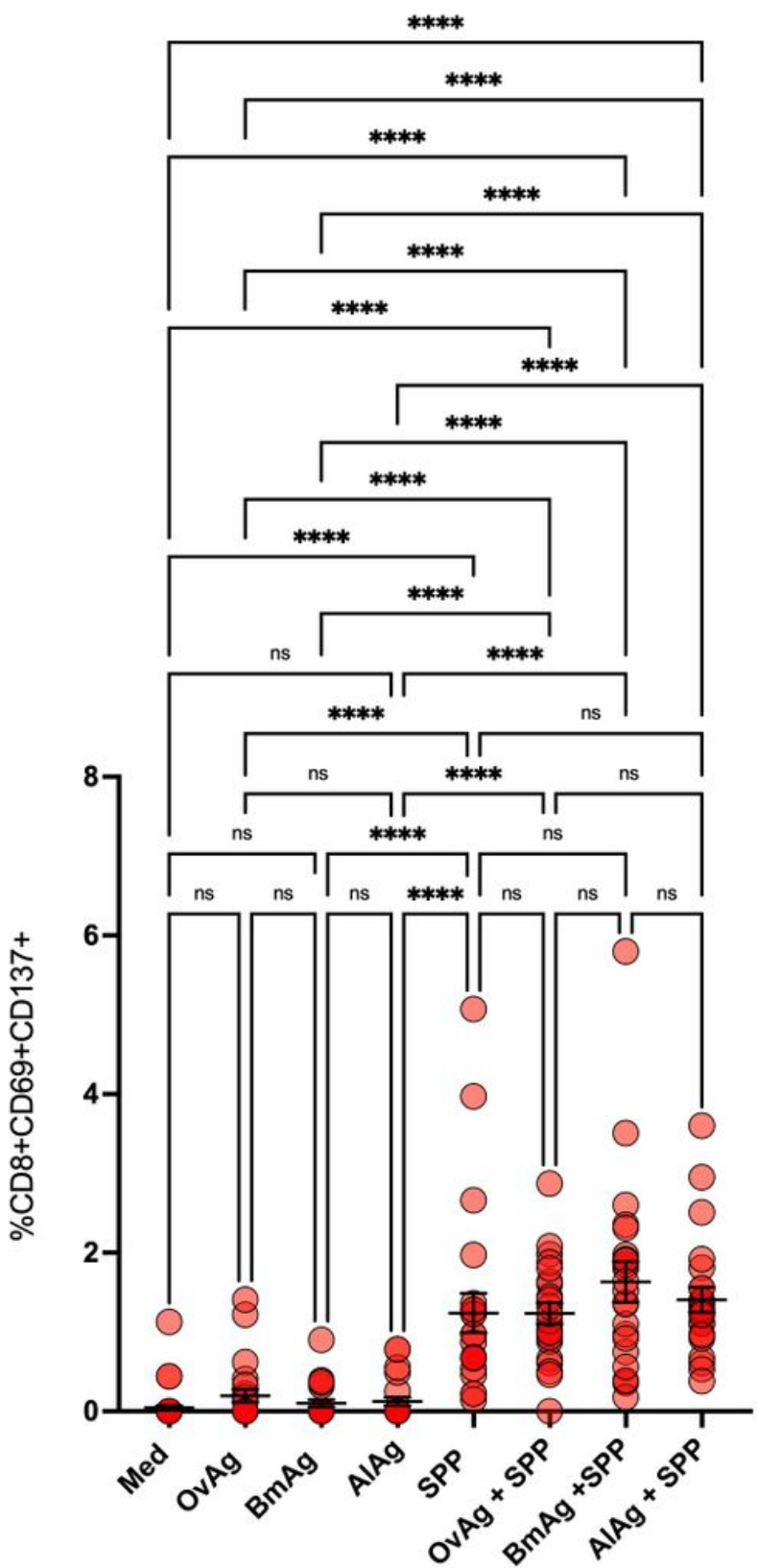

Figure 1

Modulation SARS-CoV-2-reactive CD4+ T CD8+T cells by helminth antigens in COVID-19 patients. Graphs indicate the frequency of $\mathrm{CD} 69+, \mathrm{CD} 137+$ in $\mathrm{CD} 4+(\mathrm{A})$ or $\mathrm{CD} 8+(\mathrm{B}) \mathrm{T}$ cell populations. Each symbol represents individual donors. Bars indicate Means \pm SEM of the percentage of SARS-CoV-2-reactive T cells in each setting. Data were obtained from 50 COVID-19 patients. P values were calculated using Kruskal-Wallis 'test followed by Dunn's multiple comparison post hoc. Asterisks indicate the level of 
significance: $n s=$ non-significant; $*=P \leq 0.05 ; * \star=P \leq 0.01 ; * \star *=P \leq 0.001, * \star \star *=P \leq 0.0001$. Significance is accepted if $\mathrm{P}<0.05$.

Figure 2

Suppression of SARS-CoV-2 reactive CD4+ T cells by helminth antigens correlated with an increase of IL10 production. Graphs represent cytokine levels of 3 independent experiments, and bars represent means SEM of cytokine expression - medium control. Data were obtained from 50 COVID-19 patients. Indicated $p$ values were calculated using Student's t-test. Significance is accepted if $\mathrm{P}<0.05$.

A

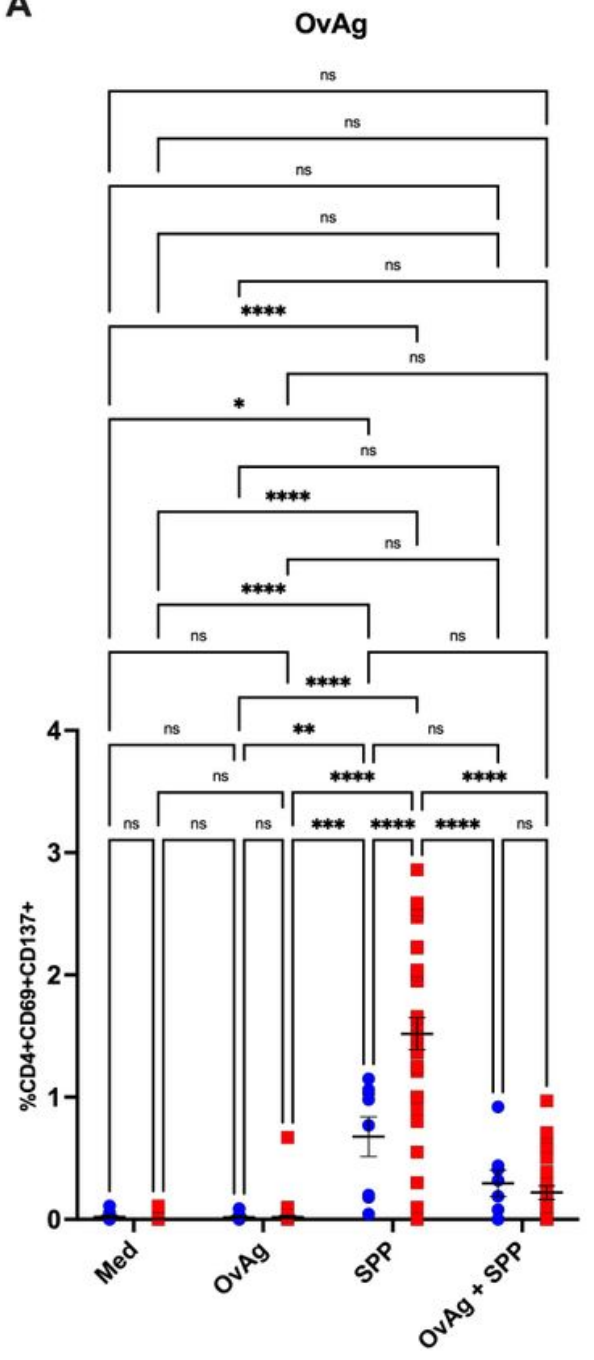

B

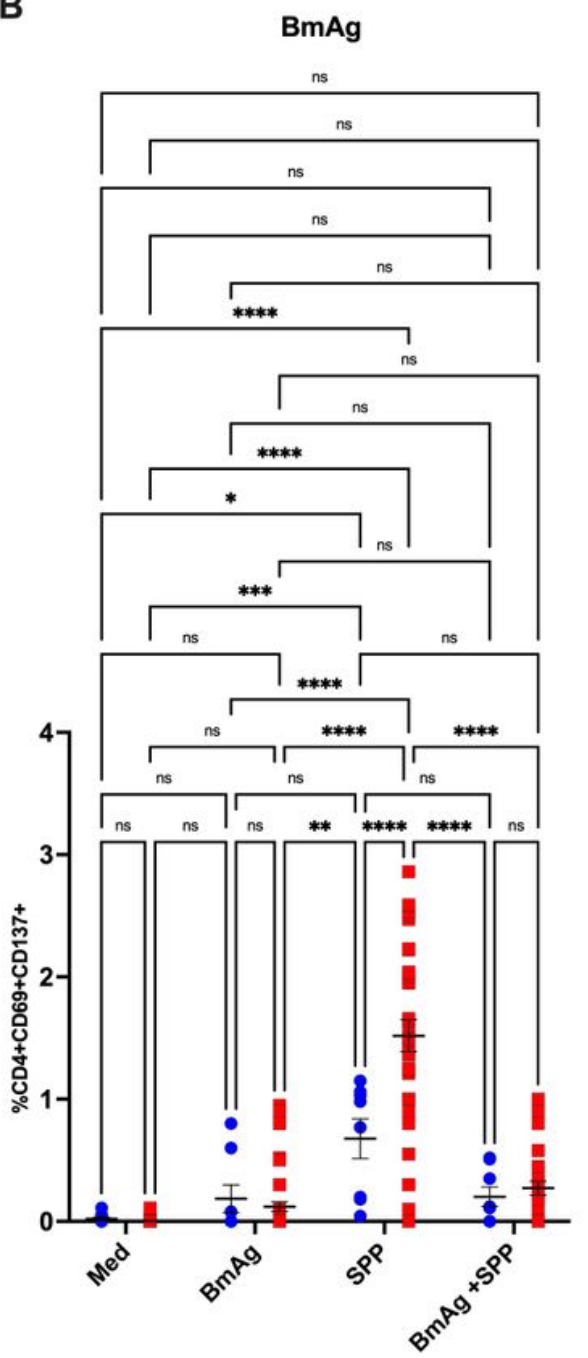

C

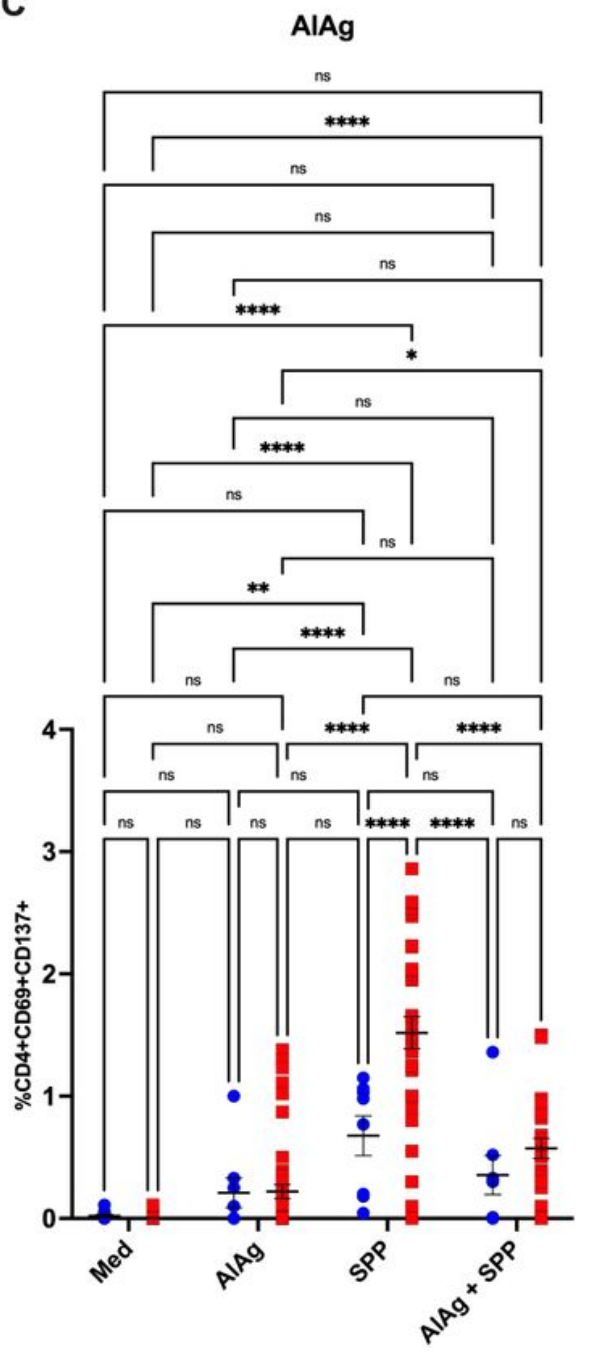

Figure 3 
Inhibition of SARS-CoV-2-reactive CD4+ by Ov, Bm, and Al antigens in healthy donors and COVID-19 patients. Graphs indicate the frequency of CD69+, CD137+ in CD4+ T cell populations. Each symbol represents individual donors. Data were obtained from 50 COVID-19 patients and 30 healthy donors. Blue circles and red squares represent the frequencies of SARS-CoV-2-reactive CD4+ cells in healthy donors and COVID-19 patients, respectively. Bars indicate Means \pm SEM of the percentage of SARS-CoV-2reactive $T$ cells in each setting. $P$ values were calculated using two-way ANOVA and Tukey's multiple comparison test. Asterisks indicate the level of significance: $n s=$ non-significant; $*=P \leq 0.05 ; * *=$ $P \leq 0.01 ; * \star *=P \leq 0.001, * \star \star \star=P \leq 0.0001$. Significance is accepted if $P<0.05$.

A

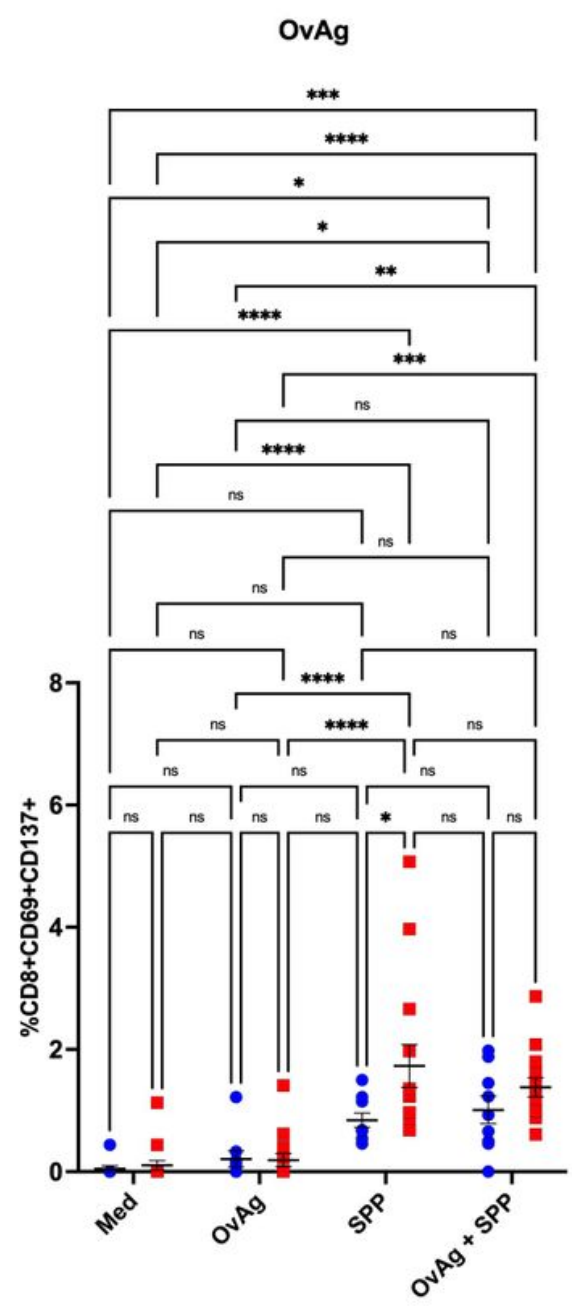

B

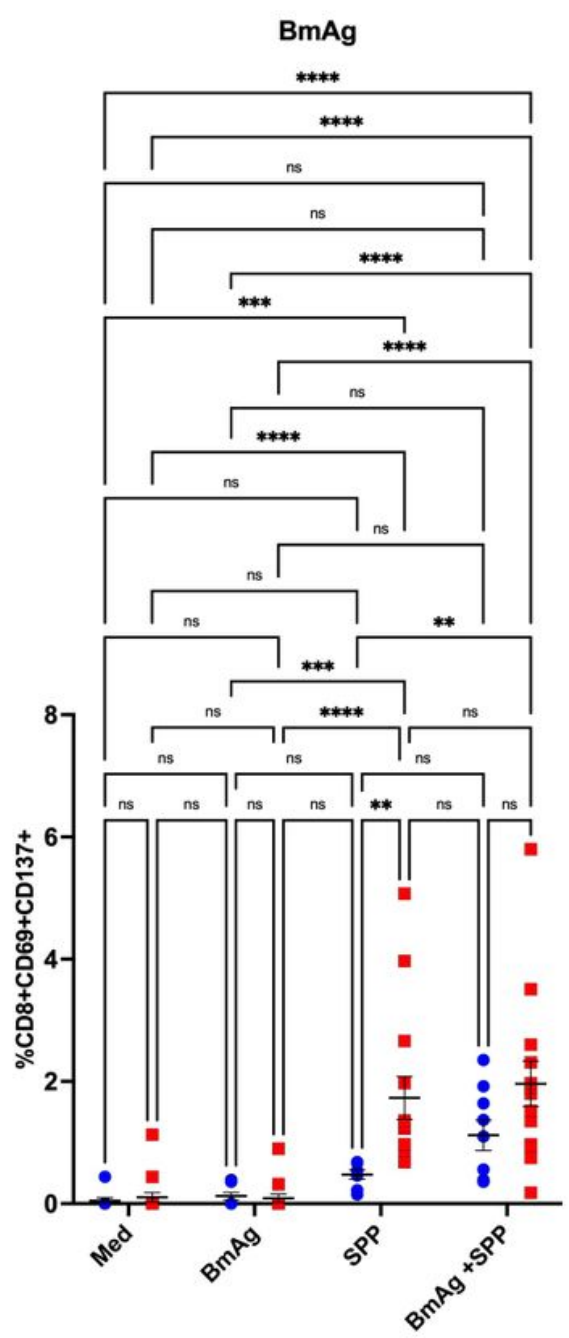

C

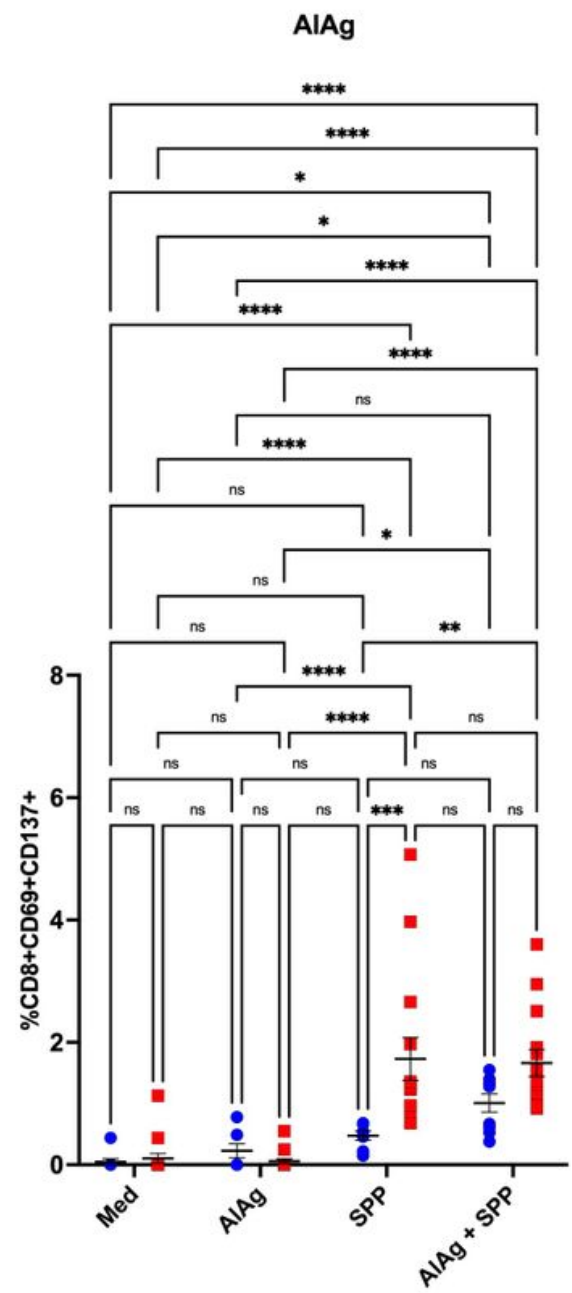

Figure 4

No suppression of SARS-CoV-2-reactive CD8+ T cells by $\mathrm{Ov}, \mathrm{Bm}$, and $\mathrm{Al}$ antigens in healthy donors and COVID-19 patients. Graphs indicate the frequency of CD69+, CD137+ in CD8+ T cell populations. Each symbol represents individual donors. Data were obtained from 50 COVID-19 patients and 30 healthy donors. Blue circles and red squares represent the frequencies of SARS-CoV-2-reactive CD8+ cells in 
healthy donors and COVID-19 patients, respectively. P values were calculated using ordinary two-way ANOVA and Tukey's multiple comparison test. Asterisks indicate the level of significance: ns= nonsignificant; ${ }^{*}=P \leq 0.05 ;{ }^{* *}=P \leq 0.01 ; * \star *=P \leq 0.001,{ }^{* \star *}=P \leq 0.0001$. Significance is accepted if $P<0.05$. 